\title{
REVISITING VARIATIONS IN TOPOLOGICAL TRANSITIVITY
}

\author{
ANIMA NAGAR
}

\begin{abstract}
Topological dynamical systems $(X, T)$ are actions $T \times X \rightarrow X$ given as $(t, x) \rightarrow t x$, on a compact, Hausdorff topological space $X$ with $T$ as an acting group or monoid. We take up the property of topological transitivity especially for semiflows $(X, S)$ and discuss the variations in its definitions. We emphasize on those properties of transitivity that differ for semiflows as compared to those for flows or (semi)cascades.
\end{abstract}

"Transitivity" gets its form from the concept of "recurrence". The frequencies of such recurrence in transitive systems were first explored and utilized by Hillel Furstenberg especially in the field of combinatorial number theory, where he used 'families' to characterize recurrence.

In his seminal paper on disjointness in topological dynamics, Furstenberg 6] laid a foundation for the classification of dynamical systems by their recurrent properties. He systematized the theory of recurrences by considering (Furstenberg) families, in particular defining such families for classifying transitivities. We follow this tradition and look into more details on various transitivities that were discussed in 2 .

Based on the study developed by Furstenberg, Akin 11 extended the notion of recurrence to the setting of general monoid actions. In this context, a 'Fursterberg family' on the subsets of an acting monoid, is considered to define the recurrence of its action.

In this article, we discuss some variations of topological transitivity for the systems $(X, T)$, on the lines of the study made in [2, infused by the ideas in [1, 6] with emphasis on the properties of "strongly transitive" systems.

For a more general approach, the survey [5] is recommended.

\section{Topological Dynamical Systems}

A pair $(X, T)$ is called a topological dynamical system, where $X$ is a compact, Hausdorff topological space and $T$ is a topological group or monoid or semigroup acting on $X$. We say that $T$ acts on $X$ when there is a continuous map $\pi: T \times X \rightarrow$ $X$ such that

(1) $\pi(e, x)=x$ for all $x \in X$, if $e \in T$ is the identity in the monoid or group $T$;

(2) $\pi(t s, x)=\pi(t, \pi(s, x))$ for all $t, s \in T$ and all $x \in X$.

$X$ is called the phase space, $T$ the acting group or monoid and the action $\pi$ gives the homeomorphism or continuous map $\pi^{t}: X \rightarrow X$ defined as $\pi^{t}(x)=t x$.

keywords: minimality, topological transitivity, strong transitivity, very strong transitivity, locally eventually onto, strongly product trantivity, semiflows

2020 Mathematical Subject Classification 37B05, 37B20

The author thanks the anonymous referees for very detailed comments and suggestions, that has tremendously polished the entire write-up. 
Remark 1.1. When $T$ is a topological group - $(X, T)$ is called a flow, and for a topological monoid $S$ - we call $(X, S)$ a semiflow. When the acting group $T=\mathbb{Z}$, then $\pi^{1}=f$ gives a generating homeomorphism on $X$, i.e. $f(x)=\pi(1, x)$, giving iterations $f^{n}(x)=\pi(n, x)=n x$. In this case we call the system $(X, f)$ a cascade. $A$ semicascade $(X, f)$ where $f: X \rightarrow X$ is a continuous mapping, corresponds to the case when the monoid $S=\mathbb{Z}_{+}=\mathbb{N} \cup\{0\}$.

In general, we call $(X, T)$ - a system without specifying if it is a flow, semiflow, cascade or semicascade. We shall specify specific cases, though our results are mostly for monoids.

Also our results remain true for any particular topology on $T$, unless we specify more assumptions on this topology.

The orbit of $x \in X$ under $T$ is $T x$, which is the smallest $T$-invariant set containing $x$. For a cascade $(X, f)$ we also call $\mathcal{O}(x)=\left\{f^{n}(x): n \in \mathbb{Z}_{+}\right\}$the (forward)orbit of the point $x$. We call $\overline{T x}$ (or $\overline{\mathcal{O}(x)}$ for cascade) the orbit closure of $x$ under $T$ and it is the smallest closed $T$-invariant set containing $x$. A set $A \subset X$ is called $T$-invariant if $T A \subset A$, where $T A=\{t a: t \in T, a \in A\}$.

For a cascade or semicascade $(X, f), x_{0} \in X$ is called a fixed point if $f\left(x_{0}\right)=x_{0}$. And $y_{0} \in X$ is called a periodic point if there exists $n \in \mathbb{N}$ such that $f^{n}\left(y_{0}\right)=y_{0}$. The smallest such $n$ is called the period of $y_{0}$.

Let $(X, T)$ and $(Y, T)$ be dynamical systems. A continuous, surjective map $\phi: X \rightarrow Y$ is called a factor-map or semi-conjugacy if $\phi(t x)=t \phi(x)$ for all $x \in X$ and $t \in T$. In addition if $\phi$ is a homeomorphism, we say that $(X, T)$ and $(Y, T)$ are conjugate as dynamical systems.

For a (semi)cascade, the $\omega$-limit set of a point $x \in X$ under $f$ is the set of all limit points of $\left\{f^{n}(x): n \in \mathbb{Z}\left(\mathbb{Z}_{+}\right)\right\}$. Thus $y \in \omega(x)$ if and only if there exists sequence $\left\{n_{k}\right\} \nearrow \infty$ such that $f^{n_{k}}(x) \rightarrow y$ or a net $\left\{n_{k}\right\}$ on some increasing directed set.

The $\omega$-limit set of a point $x \in X$ in a (semi)flow $(X, T)$, denoted as $\omega(x)$, is the set of all limit points of $\{t x: t \in T\}$. Thus we have that for $y \in \omega(x)$ and any non-empty, open $U \ni y$, the set $\{t: t x \in U\}$ is unbounded in $T$ or has no compact closure in $T$.

Remark 1.2. Observe that for all $t \in T$ and $x \in X$, we have $t \omega(x) \subset \omega(x) \subset$ $\omega(t x)$.

For very nice (say sigma-compact and locally compact) $T$, we can say that $y \in$ $\omega(x)$ if for every open $U$ with $y \in U$, there exists compact $K_{1} \subset K_{2} \subset \ldots \subset K_{i} \subset$ $\ldots \subset T$ such that $s_{i} x \in U$ for $s_{i} \in T \backslash K_{i}$, i.e. $x \in \bigcap_{s_{i} \in T \backslash K_{i}} s_{i}^{-1} U$.

We note that here we have just taken a particular representation of an "admissible set". In general such admissible sets could vary. Some of such admissible sets were first considered by Furstenberg [6].

We denote by $\mathcal{P}=\mathcal{P}(T)$ the set of all subsets of $T$. A subset $\mathcal{F} \subset \mathcal{P}$ is a Furstenberg family, if it is hereditary upwards, that is, $F_{1} \subset F_{2}$ and $F_{1} \in \mathcal{F}$ implies $F_{2} \in \mathcal{F}$. The family $\mathcal{F}$ is proper if it is a proper subset of $\mathcal{P}$. Any $\mathcal{A} \subset \mathcal{P}$ clearly generates a family $\{F \in \mathcal{P}: F \supset A$ for some $A \in \mathcal{A}\}$. A filter $\mathcal{F}$ is a proper family closed under intersection, that is, $\mathcal{F}$ is a proper subset of $\mathcal{P}$ and for $F_{1}, F_{2} \in \mathcal{F}$ implies $F_{1} \cap F_{2} \in \mathcal{F}$. The family $\mathcal{F}$ is a filter if and only if $\mathcal{F}$ is a proper family and $\mathcal{F} \cdot \mathcal{F} \subset \mathcal{F}$. A family $\mathcal{F}$ has the Ramsey property if $F \in \mathcal{F}$ and $F=F_{1} \cup F_{2}$ imply that $F_{i} \in \mathcal{F}$ for some $i \in\{1,2\}$.

For a family $\mathcal{F}$, the dual family of $\mathcal{F}$, denoted by $\mathcal{F}^{*}$, is defined as $\mathcal{F}^{*}=\{F \in$ $\mathcal{P}: F \cap F^{\prime} \neq \emptyset$, for any $\left.F^{\prime} \in \mathcal{F}\right\}$. 
For families $\mathcal{F}_{1}, \mathcal{F}_{2} \subset \mathcal{P}$, we define $\mathcal{F}_{1} \cdot \mathcal{F}_{2}=\left\{F_{1} \cap F_{2}: F_{1} \in \mathcal{F}_{1}, F_{2} \in \mathcal{F}_{2}\right\}$.

We call a family $\mathcal{F}$ to be translation invariant if $F \in \mathcal{F}$ if and only if $t^{-1}(F) \in \mathcal{F}$ (where $t^{-1}(F)$ denotes the preimage of $F$ by $t$ ), for all $t \in T$. Also $\mathcal{F}$ is thick if for $F \in \mathcal{F}$ and $t_{1}, \ldots, t_{k} \in T \Leftrightarrow \bigcap_{i=1}^{k} t_{i}^{-1}(F) \in \mathcal{F}$.

Now $F \subset T$ is called syndetic if there is a compact $K \subset T$ such that $T=K F=$ $\{k f: k \in K$ and $f \in F\}$. A set $F \subset \mathbb{Z}(\mathbb{N})$ is called syndetic if it is relatively dense, i.e., it does not contain arbitrarily large gaps.

We call a family $\mathcal{F}$ to be syndetic if every $F \in \mathcal{F}$ is syndetic.

A set $F_{1}$ is syndetic if it intersects each thick set and a set $F_{2}$ is thick if it intersects each syndetic set.

Also $F \subset T$ is called piecewise syndetic if it is an intersection of thick and syndetic subsets of $T$. We call a family $\mathcal{F}$ to be piecewise syndetic if every $F \in \mathcal{F}$ is piecewise syndetic. Thus for syndetic family $\mathcal{F}_{1} \subset \mathcal{P}$ and thick family $\mathcal{F}_{2} \subset \mathcal{P}$, the family $\mathcal{F}=\mathcal{F}_{1} \cdot \mathcal{F}_{2}$ is piecewise syndetic.

Let $\star$ be the binary operation for the group or monoid $T$. Define the finite sums of a sequence $\left\{t_{i}\right\}$ in $T$ as

$$
F S\left\{t_{i}\right\}=\left\{t_{i_{1}} \star t_{i_{2}} \star \ldots \star t_{i_{n}}: n \in \mathbb{N}\right\} .
$$

The set $F \subset T$ is called an IP set if there exists a sequence $\left\{t_{i}\right\}$ in $T$ such that $F S\left\{t_{i}\right\} \subset F$. A family $\mathcal{F}$ is called an IP family if $\mathcal{F}$ is the family of all IP sets.

We recommend the excellent treatment by Akin [1 for a detailed account on these admissible families.

Furstenberg defined families and classified different types of transitivities based on the combinatorial properties of the families of hitting sets 6].

We identify a singleton with the point it contains. For any two non-empty, open $U, V \subset X$ and $x \in X$, for the system $(X, T)$, where $T$ is any group or monoid, we define the hitting times:

$$
\begin{gathered}
N_{T}(x, V)=\{t \in T: t(x) \in V\}=\left\{t \in T: x \in t^{-1}(V)\right\} . \\
N_{T}(U, V)=\{t \in T: t(U) \cap V \neq \emptyset\}=\left\{t \in T: t^{-1}(V) \cap U \neq \emptyset\right\} . \\
N_{T}(U, x)=\{t \in T: x \in t(U)\}=\left\{t \in T: t^{-1}(x) \cap U \neq \emptyset\right\} .
\end{gathered}
$$

We see that for $x \in U, y \in V$ we have $N_{T}(x, V) \subset N_{T}(U, V)$ and $N_{T}(U, y) \subset$ $N_{T}(U, V)$.

For cascades or semicascades, we suppress the subscript $T$ from the hitting times and denote these by $N(x, V), N(U, V)$ and $N(U, x)$ and consider only positive instances as first defined in [6].

Let $S$ be any semigroup or monoid and $[S]$ denote the smallest group generated by $S$. Let $S^{-1} \subset[S]$ denote the set of inverses of elements of $S$.

We note that $N_{S}(x, V)=\{s \in S: s(x) \in V\}=\left\{s \in S: x \in s^{-1}(V)\right\}=\{s \in$ $\left.S^{-1}: x \in s(V)\right\}=N_{S^{-1}}(V, x)$. Our hitting time sets are interrelated.

We consider the product (semi)flow $\left(X^{n}, T\right)$ as $\left(x_{1}, x_{2}, \ldots, x_{n}\right) \stackrel{t}{\rightarrow}\left(t x_{1}, t x_{2}, \ldots, t x_{n}\right)$ for any $n \in \mathbb{N}$.

For each $F \in \mathcal{P}$, every point $x \in X$ and each non-empty, open $U \subset X$ define the $F$-orbit as $T^{F} x=\{t x: t \in F\}$. The $\omega$-limit set of $x$ with respect to $\mathcal{F}$, denoted by $\omega_{\mathcal{F}}(x)$, is defined as $\omega_{\mathcal{F}}(x)=\left\{y \in X: N_{T}(x, W) \in \mathcal{F}\right.$ for every open $\left.W \ni y\right\}$. 
Lemma 1.3. Let $T$ be any Abelian group or monoid. If $(y, y) \in \omega((x, y))$, then for every non-empty, open $U \ni y$, the hitting time set $N_{T}(x, U)$ is an IP set.

Proof. We prove this by induction. For non-empty, open $U \ni y$, let $U_{1}=U$ then there exists $t_{1} \in T$ such that $t_{1}(x, y) \in U_{1} \times U_{1}$. Let $U_{2}=U \cap t_{1}^{-1} U_{1}$, then since $y \in U_{2}$ there exists $t_{2} \in T$ such that $t_{2}(x, y) \in U_{2} \times U_{2}$.

Then for every $m \in F S\left\{t_{1}, t_{2}\right\}$, we have $m x \in U$.

Suppose that we get a sequence $\left\{t_{1}, t_{2}, \ldots, t_{n-1}\right\}$ in $T$ and a sequence of nonempty, open sets $U=U_{1} \supset U_{2}, \ldots, U_{n-1}$, such that for every $m \in F S\left\{t_{1}, t_{2}, \ldots t_{n-1}\right\}$, we have $m(x, y) \in U \times U$.

$$
\text { Let } U_{n}=U \cap\left(\bigcap_{m \in F S\left\{t_{1}, t_{2}, \ldots, t_{n-1}\right\}} m^{-1}(U)\right) \text {. }
$$

Then $U_{n} \ni y$ and so there exists $t_{n} \in T$ with $t_{n}(x, y) \in U_{n} \times U_{n}$.

This gives for every $m \in F S\left\{t_{1}, t_{2}, \ldots, t_{n}\right\}$, we have $m(x, y) \in U \times U$.

Thus, by induction we get a sequence $\left\{t_{i}\right\} \subset T$ for which $F S\left\{t_{i}\right\} \subset N(x, U)$.

A point $x \in X$ is said to be non-wandering in $(X, T)$ if for every neighbourhood $U$ of $x$, there is a $t \in T$ such that $t(U) \cap U \neq \emptyset$. The set of all non-wandering points of $(X, T)$ is denoted as $\Omega(T)$.

For a cascade or semicascade, a point $x \in X$ is non-wandering if for every neighbourhood $U$ of $x$ there is a $n \in \mathbb{N}$ such that $f^{n}(U) \cap U \neq \emptyset$. The set of all non-wandering points of $f$ is denoted as $\Omega(f)$.

Remark 1.4. Note that $x$ is a non-wandering point if for every open $U \subset X$ with $x \in U, N_{T}(U, U) \neq \emptyset$.

For any Furstenberg family $\mathcal{F}$, a point $x \in X$ is called an $\mathcal{F}$-non-wandering point if for every open $U \subset X$ with $x \in U$, one has $N_{T}(U, U) \in \mathcal{F}$. The set of all $\mathcal{F}$-non-wandering points, denoted by $\Omega_{\mathcal{F}}(T)$, is defined as $\Omega_{\mathcal{F}}(T)=\{x \in X$ : $N_{T}(U, U) \in \mathcal{F}$ for every open $\left.U \ni x\right\}$.

A point $x \in X$ is called recurrent whenever $x \in \omega(x)$. The set of all recurrent points of $(X, T)$ is denoted as $\mathcal{R}(X)$.

Remark 1.5. Also note that $\mathcal{R}(X)$ is $T$-invariant, i.e., $t(\mathcal{R}(X)) \subset \mathcal{R}(X)$ for all $t \in T$.

For any Furstenberg family $\mathcal{F}$, a point $x \in X$ is called $\mathcal{F}$-recurrent if and only if $x \in \omega_{\mathcal{F}}(x)$.

Definition 1.6. A point $x \in X$ is said to be strongly non-wandering in $(X, T)$ if for every neighbourhood $U$ of $x$, there is a $t \in T$ such that $x \in t(U)$. The set of all strongly non-wandering points of $(X, T)$ is denoted as $\widehat{\Omega}(T)$.

For a cascade or semicascade, a point $x \in X$ is strongly non-wandering if for every neighbourhood $U$ of $x$ there is a $n \in \mathbb{N}$ such that $x \in f^{n}(U)$. The set of all strongly non-wandering points of $f$ is denoted as $\widehat{\Omega}(f)$.

Remark 1.7. Note that $x$ is a strongly non-wandering point if for every open $U \subset X$ with $x \in U$, one has $N_{T}(U, x) \neq \emptyset$.

Remark 1.8. Note that $x$ is a strongly non-wandering point, then it is also nonwandering, i.e., $\widehat{\Omega}(T) \subset \Omega(T)$.

Definition 1.9. For any Furstenberg family $\mathcal{F}$, a point $x \in X$ is called an $\mathcal{F}$-strongly non-wandering point if for every open $U \subset X$ with $x \in U$, one has $N_{T}(U, x) \in \mathcal{F}$. The set of all $\mathcal{F}$-strongly non-wandering points, denoted by $\widehat{\Omega_{\mathcal{F}}}(T)$, is defined as $\widehat{\Omega_{\mathcal{F}}}(T)=\left\{x \in X: N_{T}(U, x) \in \mathcal{F}\right.$ for every open $\left.U \ni x\right\}$. 
Remark 1.10. Note that for systems $(X, T)$ and $(Y, S)$ and Furstenberg families $\mathcal{F}_{T} \subset \mathcal{P}(T)$ and $\mathcal{F}_{S} \subset \mathcal{P}(S)$, we have

$$
\begin{aligned}
& \Omega_{\mathcal{F}_{T}}(T) \times \Omega_{\mathcal{F}_{S}}(S) \subset \Omega_{\mathcal{F}_{T} \cdot \mathcal{F}_{S}}(T \times S), \\
& \widehat{\Omega_{\mathcal{F}_{T}}}(T) \times \widehat{\Omega_{\mathcal{F}_{S}}}(S) \subset \widehat{\Omega_{\mathcal{F}_{T} \cdot \mathcal{F}_{S}}}(T \times S),
\end{aligned}
$$

and for systems $(X, T)$ and $(Y, T)$ and Furstenberg family $\mathcal{F}=\mathcal{F}_{X}=\mathcal{F}_{Y} \subset \mathcal{P}(T)$ considering actions on $X$ and $Y$ resp., we have

$$
\begin{aligned}
& \Omega_{\mathcal{F}_{X}}(T) \times \Omega_{\mathcal{F}_{Y}}(T) \subset \Omega_{\mathcal{F}_{X} \cdot \mathcal{F}_{Y}}(T \times T), \\
& \widehat{\Omega_{\mathcal{F}_{X}}}(T) \times \widehat{\Omega_{\mathcal{F}_{Y}}}(T) \subset \widehat{\Omega_{\mathcal{F}_{X} \cdot \mathcal{F}_{Y}}}(T \times T),
\end{aligned}
$$

with equality when $\mathcal{F}$ is a filter.

We call the system $(X, T)$ to be central if each $t \in T$ is surjective, i.e., $t(X)=X$ for all $t \in T$.

The system $(X, T)$ is called $\mathcal{F}$-central for a family $\mathcal{F}$ if $N_{T}(U, U) \in \mathcal{F}$ for all $x \in X$ and open $U \ni x$. This essentially means that $\Omega_{\mathcal{F}}(T)=X$.

The system $(X, T)$ is called $\mathcal{F}$ - strongly central for a family $\mathcal{F}$ if $N_{T}(U, x) \in \mathcal{F}$ for all $x \in X$ and open $U \ni x$. This essentially means that $\widehat{\Omega_{\mathcal{F}}}(T)=X$.

A subset $S$ in an Abelian $T$ is said to be replete if $S$ contains some translate of each compact set in $T$. A set $A \subset T$ is said to be extensive if $A$ intersects every replete semigroup in $T$. A point $x \in X$ is said to be recurrent under $T$ if for every neighborhood $U \ni x$ there corresponds an extensive set $A$ in $T$ such that $A x \subset U$. This study of recurrence was first taken up by Gotschalk and Hedlund 9]. For non-Abelian groups the existence of replete semigroups seems to be quite rare, and so the definition of recurrence in these terms appears to be inadequate. But the basic idea in recurrence still keeps this structure of some admissible set of repeats of occurrences for the point that is recurrent.

We refer to [1, 6, 8, 10, 18, for more details on transformation systems.

\section{Various forms of Topological Transitivity}

2.1. Minimal Systems. Let $(X, T)$ be a flow. The simplest dynamics that one can observe is when the system is "minimal". The best treatment of minimal flows is by Auslander 3 .

A set $M \subset X$ is called a minimal set if $M$ is closed, non-empty and $T$-invariant and $M$ has no proper subset with these properties, i.e. if $N \subseteq M$ is closed and invariant then $N=M$ or $N=\emptyset$.

Remark 2.1. Note that $M \subset X$ is minimal if and only if it is the orbit closure of each of its points, i.e., $\forall x \in M$, we have $M=\overline{T x}$.

If $X=\overline{T x} \forall x \in X$, then the flow $(X, T)$ is called a minimal flow.

A point $x \in X$ is called an almost periodic point if for every neighbourhood $U$ of $x$, there is a syndetic $A \subset T$ such that $A x \subset U$.

Remark 2.2. Note that $x$ is an almost periodic point if for every open $U \subset X$ with $x \in U$, one has $N_{T}(x, U)$ is syndetic. 
We refer to 2 for more details on minimal cascades.

Noninvertible minimal semicascades were studied by Kolyada, Snoha and Trofimchuk in [13].

Example 2.3. Let $\Lambda=\{0,1\}$ and define $X=\Lambda^{\mathbb{N}}$. We consider the shift map $\sigma: X \rightarrow X$.

To obtain a minimal subset of $X$, it is enough to construct an almost periodic point $p \in X$ since then $\overline{\mathcal{O}(p)}$ will be minimal. We mimic the classical construction due to Marston Morse and Axel Thue, giving the Morse-Thue sequence.

This construction is done using substitution: $0 \rightarrow 01,1 \rightarrow 10$. Hence,

$$
0 \rightarrow 01 \rightarrow 0110 \rightarrow 01101001 \rightarrow 0110100110010110 \rightarrow \cdots
$$

This will finally converge to some $x \in\{0,1\}^{\mathbb{N}}$. This construction indicates that every finite word in $x$ occurs syndetically often.. Then $x$ is almost periodic in $\{0,1\}^{\mathbb{N}}$, and so $(\overline{\mathcal{O}(x)}, \sigma)$ is a noninvertible minimal dynamical system.

A map $f: X \rightarrow X$ is called almost open if for every non-empty open subset $U \subset X$, the set $f(U)$ has non-empty interior in $X$. Essentially a map is almost open if and only if the inverse image of every dense subset is dense. A map $f: X \rightarrow Y$ is called irreducible if the only closed $A \subset X$ for which $f(A)=Y$ is $A=X$. In particular, an irreducible map is always surjective. Also, irreducible maps are always almost open. Minimal maps are irreducible, and irreducible maps are almost open; thus implying minimal semicascades to be almost open.

Remark 2.4. We note that irreducibility or almost openess does not imply minimality even for cascades as can be seen in the below example.

Let $X=\mathbb{T} \times\{1,2\}$ where $T$ is the unit circle bijective with $[0,1)$ and consider the irrational rotation on both circles in $X$ given as $\tau(\theta, i)=(\alpha \theta, i)$ for $i=1,2$. Then $(X, \tau)$ is irreducible, almost open but not minimal.

For more on properties of minimal semicascades we refer to [13].

This leads to the natural question on the properties of minimal semiflows.

We note that recently such a study is done by Auslander and Dai [4]. However, they have a different perspective.

Definition 2.5. A minimal semiflow is the system $(X, S)$, where $S$ is a semigroup or monoid action on $X$, for which $X=\overline{S x}$ is true $\forall x \in X$.

Example 2.6. Consider $X=\mathbb{T}$, the unit circle bijective with $[0,1)$ and consider

$$
S=\left\{f_{p, q}(x):=p x+q(\bmod 1): p \in \mathbb{Q} \cap(0,1], q \in \mathbb{Q} \cap[0,1]\right\}
$$

with operation as composition of functions. Then $S$ is an equicontinuous family in $\mathcal{C}(X)$ (the space of all continuous real valued functions on $X$ with the uniform topology), and each $f_{p, 0} \in S$ is invertible but $f_{p, 0}^{-1} \notin S$ for all $p \in(0,1)$. Also the monoid $S$ acts on $X$ and $(X, S)$ is a semiflow.

For the semiflow $(X, S)$, we note that $\overline{S x} \supset \overline{\left\{f_{1, q}(x): q \in[0,1] \cap \mathbb{Q}\right\}}=X$ for all $x \in X$ and so $(X, S)$ is minimal.

Let $0 \in X$ and open $U \ni 0$ and let $A=\left\{f_{r, s} \in S: f_{r, s}(0) \in U\right\}=N_{S}(0, U)$.

Note that if $A$ is syndetic then there would exist compact $K \subset S$ such that $S=K A$.

Observe that if $U=\left[0, \frac{1}{27}\right) \cup\left(\frac{26}{27}, 1\right]$ with 0 identified with 1 , then here 


$$
A=\left\{f_{r, s}: r \in(0,1] \cap \mathbb{Q}, s \in\left(\left[0, \frac{1}{27}\right) \cup\left(\frac{26}{27}, 1\right]\right) \cap \mathbb{Q}\right\} .
$$

Let $h=\frac{1}{81}$ then $f_{h, 0} S \subset A$. But $f_{h, 0}^{-1} \notin S$ and so we cannot have $S=f_{h, 0}^{-1} A$.

If there is a compact $K \subset S$ such that $S=K A$ then for all $s \in \mathbb{Q} \cap[0,1]$, there exists $f_{1, k} \in K$ and $f_{1, a} \in A$ such that $f_{1, s}=f_{1, k} f_{1, a}$, i.e. $s=k+a$ for all $s \in \mathbb{Q} \cap[0,1]$. Since $f_{1, a} \in A$ only for $a \in\left(\left[0, \frac{1}{27}\right) \cup\left(\frac{26}{27}, 1\right]\right) \cap \mathbb{Q}$, we must have $f_{1, t} \in K$, say for all $t \in\left[\frac{5}{27}, \frac{13}{27}\right] \cap \mathbb{Q}$. Let $i \in\left[\frac{5}{27}, \frac{13}{27}\right] \cap(\mathbb{R} \backslash \mathbb{Q})$ and let $r_{n} \in\left[\frac{5}{27}, \frac{13}{27}\right] \cap \mathbb{Q}$ be such that $r_{n} \rightarrow i$. If $K$ is compact, $\left\{f_{1, r_{n}}\right\}$ must uniformly converge in $K$. This is not possible.

Thus $N_{S}(0, U)$ is not syndetic.

Remark 2.7. For a minimal semiflow $(X, S)$, each $s \in S$ need not be surjective, nor almost one-to-one, nor $N_{S}(x, U)$ be syndetic for every open $U \subset X$ with $x \in U$.

However, Zorn's lemma guarantees that every semiflow will have a minimal closed invariant subset.

Remark 2.8. We note that in the example above, every $s \in S$ is not surjective. Hence though every point of $X$ is recurrent, the system is not distal. In fact the semiflow $(X, S)$ is proximal.

We note here that if $[S]$ denote the smallest group generated by the monoid $S$, then $(X, S)$ proximal implies that $(X,[S])$ is also proximal. However the converse here may not be true. For example we can consider $S^{-1}$ in Example 2.6. Note that $[S]=\left\{f_{a, b}:=a x+b: a \in(0, \infty) \cap \mathbb{Q}, b \in \mathbb{Q}\right\}$ here.

Remark 2.9. It has been observed in [7] that the only endomorphism a minimal proximal flow admits is the identity automorphism. We note that minimal proximal semiflows also admit the identity automorphism as the only endomorphism, and the proof is similar as the one for flows.

However, every minimal proximal flow is weakly mixing [7] whereas Example 2.6] is a minimal proximal semiflow that is not weakly mixing. In fact, it is equicontinuous.

We summarize the trivial properties of minimal sets in semiflows:

Proposition 2.10. For the semiflow $(X, S)$ :

(1) If $M_{1}$ and $M_{2}$ are minimal subsets of $X$ for any semiflow $(X, S)$ then either $M_{1}=M_{2}$ or $M_{1} \cap M_{2}=\emptyset$.

(2) Let $(X, S)$ be a semiflow. Then $X$ contains a minimal set.

(3) If $(X, S)$ is minimal then the only closed, invariant subsets of $X$ are $\emptyset$ and $X$.

(4) For semiflows $(X, S)$ and $(Y, S)$, let $\pi: X \rightarrow Y$ be a semi-conjugacy.

Then if $X_{0} \subset X$ is minimal then $\pi\left(X_{0}\right)=Y_{0} \subset Y$ is minimal.

Definition 2.11. Consider a semiflow $(X, S)$. The semiflow $(X, S)$ is irreducible if every $s \in S$ is irreducible (according to the definition in [13]).

We note that the image of no proper closed subset of $X$ under the action of the semigroup $S$ can be equal to $X$ in an irreducible semiflow. Also when $(X, S)$ is irreducible then it is also central.

Remark 2.12. We note that in the Example [2.6, the semiflow $(X, S)$ is not irreducible. Thus for semiflows, minimality does not imply irreducibility. 
Definition 2.13. Consider a semiflow $(X, S)$. Then $s \in S$ is almost open if $s U$ has a non-empty interior for all non-empty, open $U \subset X$. Equivalently $s^{-1}(D)$ is dense in $X$ whenever $D$ is dense in $X$. The semiflow $(X, S)$ is almost open if each $s \in S$ is almost open.

The semiflow $(X, S)$ is open if each $s \in S$ is an open map.

Remark 2.14. We note that in Example [2.6, all the elements in $S$ are almost open. Thus there are semiflows that are not irreducibe but almost open.

Proposition 2.15. Let a semiflow $(X, S)$ be minimal and $U \subset X$ be non-empty and open. Then there exists $s_{1}, \ldots, s_{m} \in S$ such that $X=\bigcup_{j=1}^{m} s_{j}{ }^{-1}(U)$.

Furthermore, if $S$ is Abelian and also central, then there exists $s_{n_{1}}, \ldots, s_{n_{m}} \in S$ such that $X=\bigcup_{j=1}^{m} s_{n_{j}}(U)$.

Proof. Since $(X, S)$ is minimal, $\overline{S x}=X$ for all $x \in X$. Consider a non-empty, open set $U \subset X$. For every $x \in X$, there exists $s \in S$ such that $s(x) \in U$. And so we have open neighbourhood $V_{x} \ni x$ with $V_{x} \subset s^{-1}(U)$. Then the class of open sets $\left\{V_{x}: \quad x \in X\right\}$ forms an open cover of $X$. By compactness of $X$, this open cover has a finite sub-cover and so $X=\bigcup_{j=1}^{m} V_{x_{j}}$. Also corresponding to each $x_{j}$ there is an $s_{j} \in S$ such that $V_{x_{j}} \subset s_{j}^{-1}(U)$. And so $X=\bigcup_{j=1}^{m} V_{x_{j}} \subset \bigcup_{j=1}^{m} s_{j}{ }^{-1}(U) \subset X$.

Furthermore when $S$ be Abelian with each $s \in S$ surjective, then $s(X)=X$, $\forall s \in S$. Now $X=\bigcup_{j=1}^{m} s_{j}{ }^{-1}(U)$. Then for $s=s_{1} \circ \ldots \circ s_{m}$ if $s_{n_{j}}=s s_{j}{ }^{-1}$,

$$
X=s(X)=\bigcup_{j=1}^{m} s s_{j}{ }^{-1}(U)=\bigcup_{j=1}^{m} s_{n_{j}}(U) .
$$

Corollary 2.16. Let a semiflow $(X, S)$ be minimal and irreducible with $S$ Abelian. Then for all non-empty, open $U \subset X$ then there exists $s_{n_{1}}, \ldots, s_{n_{m}} \in S$ such that $X=\bigcup_{j=1}^{m} s_{n_{j}}(U)$.

2.2. Topological Transitivity. Topological transitivity can be described as the eventuality of neighbourhood of every point to visit every region of the phase space at some time.

A flow or semiflow $(X, T)$ is called topologically transitive or transitive if for all non-empty open sets $U, V \subset X$, there is a $t \in T$ for which $t(U) \cap V \neq \emptyset$ [Equivalently, $\left.U \cap t^{-1}(V) \neq \emptyset\right]$.

A cascade or semicascade $(X, f)$ is said to be topologically transitive if for every pair of non-empty open sets $U, V$ in $X$, there is a $n \in \mathbb{N}$ such that $f^{n}(U) \cap V \neq \emptyset$ [Equivalently, $U \cap f^{-n}(V) \neq \emptyset$ ].

A flow or semiflow $(X, T)$ is called point transitive if there is an $x_{0} \in X$ such that $\overline{T x_{0}}=X$ ( i.e. $\left\{t x_{0}: t \in T\right\}$ is dense in $X$ ).

An $x \in X$ is called a transitive point, if $\overline{T x}=X$. We note that $x$ is transitive when $\omega(x) \cup T x=\mathrm{X}$.

We denote the set of all transitive points of $(X, T)$ by $\operatorname{Trans}(T)$.

Remark 2.17. We note that for a minimal system $\operatorname{Trans}(T)=X$. 
The cascade or semicascade $(X, f)$ is said to be point transitive if there is an $x_{0} \in X$ such that $\overline{\mathcal{O}\left(x_{0}\right)}=X$ (i.e. $X$ has a dense orbit). All such points with dense orbits are called transitive points and the set of transitive points in $X$ is denoted as $\operatorname{Trans}(f)$.

Both these definitions of point transitivity and topological transitivity for (semi)cascades are equivalent, in a wide class of spaces, including all perfect, compact metric spaces. We recall,

Proposition 2.18. 12] For a cascade or semicascade $(X, f)$, if $X$ has no isolated point then point transivity implies the transitivity of $(X, f)$.

The converse holds if $X$ is separable and of second category.

Though we see that this is not essentially true for a semiflow $(X, S)$ when $S$ is not discrete.

Example 2.19. Let $S=\left(\mathbb{R}^{+},+\right)$be the monoid of nonnegative real numbers and $X=[0, \infty]$, the one-point compactification of the nonnegative reals. Define $S \times X \rightarrow X$, by $(s, x) \rightarrow s+x$. Then for the natural action of $S$ on $X,(X, S)$ is a semiflow.

Then for the semiflow $(X, S), \overline{S 0}$ is dense in $X$ and so $\operatorname{Trans}(S) \neq \emptyset$. Thus $(X, S)$ is point transitive but it can be clearly seen that $(X, S)$ is not topologically transitive.

Remark 2.20. We note this obvious fact: if $(X, S)$ is minimal, then $(X, S)$ is topologically transitive.

The proof of the following follows trivially from known facts.

Proposition 2.21. We have the following equivalent conditions for transitivity of semiflows $(X, S)$ :

(1) $(X, S)$ is topologically transitive.

(2) for every pair of non-empty open sets $U$ and $V$ in $X$, there is a $s \in S$ such that $s^{-1}(U) \cap V \neq \emptyset$.

(3) for every pair of non-empty open sets $U$ and $V$ in $X, N_{S}(U, V) \neq \emptyset$.

(4) for every non-empty open set $U \subset X$, the set $\cup_{s \in S} s(U)$ is dense in $X$.

(5) for every non-empty open set $U \subset X$, the set $\cup_{s \in S} s^{-1}(U)$ is dense in $X$.

(6) if $E \subset X$ is closed and $S E \subset E$, then either $E=X$ or $E$ is nowhere dense in $X$.

(7) if $U \subset X$ is open and $U \subset S U$, then either $U=\emptyset$ or $U$ is dense in $X$.

Moreover, if $X$ is a compact, perfect metric space, then

(1) There exists $x \in X$ such that the orbit $S x$ is dense in $X, i$. e. the set Trans $(S)$ of transitive points is non-empty.

(2) The set Trans $(S)$ of transitive points equals $\{x: \omega(x)=X\}$ and it is a dense subset of $X$.

Example 2.22. Let $f:[0,1] \rightarrow[0,1]$ be the tent map defined as $f(x)= \begin{cases}2 x, & 0 \leq x \leq 1 / 2 ; \\ 2(1-x), & 1 / 2 \leq x \leq 1\end{cases}$

Then the semicascade $([0,1], f)$ is transitive.

Here for any non-empty open $J$ in $[0,1], \exists n \in \mathbb{N}$ such that $f^{n}(J)=[0,1]$. Thus $([0,1], f)$ is transitive.

Remark 2.23. Any transitive homeomorphism is irreducible, almost open and almost one-to-one. 
For more on transitivity, we refer to [2, 10, 12, 17.

For a Furstenberg family in $\mathbb{N}, \mathcal{F}$-point transitivity was first defined by Jian $\mathbf{L i}$ in [15] for (semi)cascades. In general, $\mathcal{F}$-transitivity does not imply $\mathcal{F}$-point transitivity.

We look into this concept for (semi)flows. Let $\mathcal{F}$ be a Furstenberg family of subsets of $T$. A point $x \in X$ is called an $\mathcal{F}$-transitive point if for every non-empty, open $U \subset X$, one has $N_{T}(x, U) \in \mathcal{F}$.

The (semi)flow $(X, T)$ is called $\mathcal{F}$-point transitive if there exists some $\mathcal{F}$-transitive point $x_{0} \in X$.

For the Furstenberg family $\mathcal{F}$, the (semi)flow $(X, T)$ is called $\mathcal{F}$-transitive if for every pair $U, V$ of non-empty, open sets in $X$, one has $N_{T}(U, V) \in \mathcal{F}$.

Remark 2.24. Note that if $(X, T)$ is $\mathcal{F}$-transitive then $(X, T)$ is also $\mathcal{F}$-central and $\Omega_{\mathcal{F}}(T)=X$ though it need not be $\mathcal{F}$-point transitive.

We recommend the enthusiastic reader to look into a detailed description of $\mathcal{F}$-transitivity discussed by Akin in [1].

2.3. Weakly Mixing and (Strongly) Mixing. A (semi)flow $(X, T)$ is said to be weakly mixing if the product (semi)flow $\left(X^{2}, T\right)$ is transitive. $(X, T)$ is called (strong) mixing if for every pair $V, W$ of non-empty open sets in $X$, there is a compact $K \subset T$ such that $t(V) \cap W$ is non-empty for all $t \in T \backslash K$.

A (semi)cascade $(X, f)$ is said to be weakly mixing if the product system $(X \times$ $X, f \times f)$ is transitive. $(X, f)$ is called mixing if for every pair $V, W$ of non-empty open sets in $X$, there is a $N>0$ such that $f^{n}(V) \cap W$ is non-empty for all $n \geq N$.

Remark 2.25. If $(X, T)[(X, f)]$ is mixing then it is weakly mixing.

Remark 2.26. Note that $(X, T)$ is mixing if and only if for every pair of nonempty, open sets $U, V \subset X$ the set $N_{T}(U, V)=T \backslash K$, for some compact $K \subset T$. The cascade $(X, f)$ is mixing if and only if for every pair of non-empty, open sets $U, V \subset X, N(U, V)$ is cofinite.

Remark 2.27. Note that for any non-empty, open $U_{1}, U_{2}, V_{1}, V_{2} \subset X$, we have $N_{T}\left(U_{1} \times V_{1}, U_{2} \times V_{2}\right)=N_{T}\left(U_{1}, U_{2}\right) \cap N_{T}\left(V_{1}, V_{2}\right)$.

This observation enables us to say that for a (semi)flow $(X, T)$,

Proposition 2.28. [1] The following are equivalent.

(1) $(X, T)$ is weak mixing.

(2) For non-empty, open sets $U_{1}, V_{1}, U_{2}, V_{2} \subset X$, there exists a $t \in T$ such that $t U_{1} \cap V_{1} \neq \emptyset$ and $t U_{2} \cap V_{2} \neq \emptyset$.

(3) For non-empty, open sets $U_{1}, V_{1}, U_{2}, V_{2} \subset X$, there exists a $t \in T$ such that $t^{-1} U_{1} \cap V_{1} \neq \emptyset$ and $t^{-1} U_{2} \cap V_{2} \neq \emptyset$.

(4) The collection $\left\{N_{T}(U, V)\right.$ : for $U, V$ non-empty, open in $\left.X\right\}$ has the finite intersection property (or equivalently it generates a filter of subsets of $T$ ).

Further, if $T$ is Abelian then for every $N \in \mathbb{N}$ the product system $\left(X^{N}, T\right)$ is topologically transitive.

Note that for Abelian $T$ the product system $\left(X^{N}, T\right)$ being topologically transitive is a well-known consequence of the Furstenberg Intersection Lemma.

Lemma 2.29 (Furstenberg Intersection Lemma [1]). For a system $(X, T)$, with $T$ Abelian, assume that $N_{T}(U, V) \cap N_{T}(U, U) \neq \emptyset$ for every pair of non-empty, open 
$U, V \subset X$. Then for all non-empty, open $U_{1}, V_{1}, U_{2}, V_{2} \subset X$ there exist non-empty, open $U_{3}, V_{3} \subset X$ such that

$$
N_{T}\left(U_{3}, V_{3}\right) \subset N_{T}\left(U_{1}, V_{1}\right) \cap N_{T}\left(U_{2}, V_{2}\right) .
$$

The concept of weakly mixing was first defined by Furstenberg in [6], who showed that the (semi)cascade $(X, f)$ is weakly mixing if each $N(U, V)$ is thick, and is mixing if each $N(U, V)$ is cofinite; for non-empty, open subsets $U, V$ of $X$.

Proposition 2.30. For a metric semicascade $(X, f)$, where $X$ is a compact and perfect, the semicascade is weakly mixing if and only if there exists a sequence $\left(x_{1}, x_{2}, \ldots\right) \in X^{\infty}$ such that for all $n \in \mathbb{N},\left(x_{1}, \ldots, x_{n}\right)$ is a transitive point for $\left(X^{n}, f^{(n)}\right)$.

We call such a sequence a strongly transitive sequence.

Proof. The semicascade $(X, f)$ is weakly mixing if and only if $\left(X^{n}, f^{(n)}\right)$ is transitive for every $n \in \mathbb{N}$ if and only if there exists $\left(x_{1}, \ldots, x_{n}\right) \in X^{n}$ that is a transitive point for $\left(X^{n}, f^{(n)}\right)$.

We note that since $\left(X^{n} \times X, f^{(n)} \times f\right)$ is transitive, the transitive point $\left(x_{1}, \ldots, x_{n}\right)$ for $\left(X^{n}, f^{(n)}\right)$ gives $\left(x_{1}, \ldots, x_{n}, x_{n+1}\right) \in X^{n+1}$ which is a transitive point for $\left(X^{n+1}, f^{(n+1)}\right)$. Thus the result follows by induction.

Remark 2.31. The existence of strongly transitive sequence is equivalent to the system being weakly mixing. This is similar to the concept of independent set due to Iwanik [1].

For more on weakly mixing and mixing, we refer to [2, 6, 8, 10, 18.

The (semi)flow $(X, T)$ is weakly mixing if for all $U, V$ non-empty, open in $X$, $N_{T}(U, V)$ is thick. And is mixing if for all $U, V$ non-empty, open in $X, N_{T}(U, V)$ is co-compact (complement of a compact subset of $T$ ).

Let $\mathcal{F}$ be a Furstenberg family. Then $(X, T)$ is $\mathcal{F}$-weakly mixing if $X \times X$ is $\mathcal{F}$-transitive if and only if $\mathcal{F}$ has the finite intersection property if and only if $\mathcal{F}$ is thick.

A more detailed description on this can be seen in [1].

We recall the concept of $\mathcal{F}$-point transitive from the previous section. In particular for cascades or semicascades $(X, f)$, we have;

Proposition 2.32. [15] If $(X, f)$ is weakly mixing then $(X, f)$ is $\mathcal{F}$-point transitive with $\mathcal{F}$ an IP family in $\mathbb{Z}_{+}$.

We note that this can be proved similarly, in general, for any flow or semiflow.

Proposition 2.33. For Abelian $T$, if $(X, T)$ is weakly mixing then $(X, T)$ is $\mathcal{F}$-point transitive with $\mathcal{F}$ an IP family in $T$.

Using Lemma 1.3 the proof follows similarly as in 15 and we skip it.

Definition 2.34. Let $\mathcal{F}$ be a Furstenberg family such that $\mathcal{F} \cdot \mathcal{F}=\mathcal{F}$. A sequence $\left(x_{1}, x_{2}, \ldots\right) \in X^{\infty}$ is called $\mathcal{F}$-strongly transitive sequence if for all $n \in \mathbb{N}$, $\left(x_{1}, \ldots, x_{n}\right)$ is an $\mathcal{F}$ - transitive point for $\left(X^{n}, T\right)$.

We have an analogue to Proposition 2.30.

Proposition 2.35. For Abelian $T$ and $X$ a compact and perfect metric space, if the system $(X, T)$ is weakly mixing then there exists a $\mathcal{F}$-strongly transitive sequence $\left(x_{1}, x_{2}, \ldots\right) \in X^{\infty}$ with $\mathcal{F}$ an IP family in $T$. 


\subsection{Locally Eventually ONTO.}

Definition 2.36. A semiflow $(X, S)$ is called locally eventually onto when for any non-empty, open $U \subset X$ there exists a compact $K \subset S$ such that $s(U)=X$, $\forall s \in S \backslash K$.

A semicascade $(X, f)$ is locally eventually onto if and only if for any nonempty, open $U \subset X$ there exists $N \in \mathbb{N}$ such that $f^{N}(U)=X$.

Remark 2.37. We note that for a homeomorphism $h$, it is impossible for a proper, non-empty open $U \subset X$ to satisfy $h(U)=X$. Thus, no flows or cascades can be locally eventually onto.

Recall Example 2.22 of the tent-map. This is an example of a locally eventually onto semicascade.

Also Example 2.50 is an example of a locally eventually onto semiflow.

Recall that $(X, f)$ is locally eventually onto then $N(U, x)$ is co-finite in $\mathbb{N}$ for all non-empty, open $U \subset X$ and all $x \in X$.

We recommend [2], and the references therein, for more details on locally eventually onto semicascades.

Proposition 2.38. We have for a metric semiflow $(X, S)$, the following to be equivalent.

(1) The semiflow is locally eventually onto.

(2) For all $\epsilon>0$, there exists a compact $K \subset T$ such that $t^{-1}(x)$ is $\epsilon$-dense in $X$ for every $x \in X$ and $\forall t \in T \backslash K$.

We note that for a locally eventually onto semiflow $(X, S)$, for every non-empty, open $U \subset X$, there exists a compact $H \subset T$ such that $\bigcup_{h \in H} h U=X$.

Corollary 2.39. A locally eventually onto system is always mixing and hence weakly mixing and thus transitive.

Example 2.40. Recall the example of the full 2-shift. It is mixing but fails to be locally eventually onto, as observed in [2].

$(X, S)$ is locally eventually onto then $N_{S}(U, x)$ is co-compact in $S$, i.e. with compact complement, for all non-empty, open $U \subset X$ and all $x \in X$.

Remark 2.41. A locally eventually onto system need not be almost open, as observed in 2 .

2.5. Strongly Transitive \& Very Strongly Transitive. The concept of transitivity deals with denseness of some orbit, while the concept of minimality implies that every orbit is dense. What would result if we want every "backward orbit" to be dense? We recall a few basics on this from 2, 17, and discuss further details on this.

For the cascade or semicascade $(X, f)$, the backward(negative) orbit of $x \in X$ is denoted as $\mathcal{O}^{-}(x)$ and defined as,

$$
\mathcal{O}^{-}(x)=\left\{y \in X: f^{n}(y)=x \text { for some } n \in \mathbb{N}\right\}
$$

A cascade or semicascade $(X, f)$ is strongly transitive if $X=\bigcup_{n \in \mathbb{N}} f^{n}(U)$ for every non-empty, open $U \subset X$.

Let $(X, f)$ be any system, then $f$ is called iteratively almost open if for every non-empty, open $U \subset X f^{n}(U)^{\circ} \neq \emptyset$ for infinitely many $n \in \mathbb{N}$. 
Recall that if $(X, f)$ is strongly transitive, then $f$ is iteratively almost open 22.

$(X, f)$ is called Very Strongly Transitive (VST) if for every non-empty, open $U \subset X$ there is a $N \in \mathbb{N}$ such that $\bigcup_{n=1}^{N} f^{n}(U)=X$.

Recall that if $(X, f)$ is very strongly transitive then for every non-empty, open set $U \subset X$ and every point $x \in X$, the set $N(U, x)$ is syndetic and for any nonempty, open $U, V \subset X$, the set $N(U, V)$ is syndetic. And that if $f$ is an open map then $(X, f)$ is very strongly transitive if and only if $(X, f)$ is strongly transitive [2].

We refer to [2] for many other properties of strongly transitive and very strongly transitive semicascades.

What can we say about this concept for some flow $(X, T)$ ?

Now $T^{-1}(x)=\{y \in X: t(y)=x$ for some $t \in T\}$ is the same as $T x$. And so the concept that every backward orbit is dense is same as the concept that every orbit is dense which is the same as minimality. So thinking about this concept for group actions gives nothing new. It just means minimality. This is also observed in [2] - for cascades strongly transitive is equivalent to being minimal, and is a distinct property only for semicascades.

So do we get anything new if we look into semigroup actions?

Let $S$ be a semigroup and consider the semiflow $(X, S)$. Define $S^{-}(x)=\{y \in$ $X: s(y)=x$ for some $s \in S\}$.

Definition 2.42. We call a semiflow $(X, S)$ strongly transitive if $X=\bigcup_{s \in S} s U$, for every non-empty, open set $U \subset X$.

We call a semiflow $(X, S)$ very strongly transitive if for every non-empty, open set $U \subset X$ there exists a compact $K \subset S$ for which $X=\bigcup_{s \in K} s U$.

Proposition 2.43. For a semiflow $(X, S)$ the following are equivalent:

(1) $(X, S)$ is strongly transitive.

(2) For every non-empty, open set $U \subset X$ and every point $x \in X$, there exists $s \in S$ such that $x \in s(U)$.

(3) For every non-empty, open set $U \subset X$ and every point $x \in X$, the hitting set $N_{S}(U, x)$ is non-empty.

(4) $S^{-}(x)$ is dense in $X$ for every $x \in X$.

This further implies that each point in $X$ is strongly non-wandering i.e. $\widehat{\Omega}(S)=$ $X$.

Also, if $(X, S)$ is strongly transitive, then $(X, S)$ is topologically transitive.

Proposition 2.44. If $(X, S)$ is very strongly transitive then

(1) for every non-empty, open set $U \subset X$ and every point $x \in X$, the hitting set $N_{S}(U, x)$ is syndetic, i.e. there exists a compact $K \subset S$ such that $S=K N_{S}(U, x)$.

(2) for any non-empty, open $U, V \subset X$, the set $N_{S}(U, V)$ is syndetic, i.e. there exists a compact $K \subset S$ such that $S=K N_{S}(U, V)$.

Further, if $(X, S)$ is very strongly transitive then it is also strongly transitive.

Recall the definition of open semiflows from Definition 2.13

Proposition 2.45. If semiflow $(X, S)$ is open then the two concepts of strongly transitive and very strongly transitive are the same.

We skip the trivial proofs here.

Remark 2.46. The concepts of strongly transitive and very strongly transitive are distinct for semicascades, as can be seen from the example in [2]. 
Remark 2.47. For semiflow $(X, S)$, by Proposition 2.15 minimality implies very strongly transitive and hence strongly transitive when $S$ is Abelian and central.

Remark 2.48. For semiflow $(X, S)$, by Theorem 2.38 locally eventually onto implies very strongly transitive and hence strongly transitive.

We note that strongly transitive neither implies nor is implied by minimality in case of semiflows. Consider the examples:

Example 2.49. Consider $X=\mathbb{R} \cup\{\infty\}$, the one-point compactification of the reals. For every $r \in \mathbb{R}$ define $f_{r}: X \rightarrow X$ as $f_{r}(y)=y+r, \forall y \in X$. Then $S=\left\{f_{r}: r \in \mathbb{R}\right\}$ is an equicontinuous family in $\mathcal{C}(X)$ (the space of all continuous real valued functions on $X$ with the uniform topology), which is a semigroup with the operation of composition of functions.

For the semiflow $(X, S)$, one has $\overline{S x}=X$ for all $x \in X$ and so $(X, S)$ is minimal.

However, $S^{-}(\infty)=\emptyset$ and so $(X, S)$ is not strongly transitive.

Example 2.50. Let $X=\mathbb{T}$, and consider the semigroup $S=\mathbb{R}^{+}$of all nonnegative real numbers, with the operation addition. Consider the action $\pi: S \times X \rightarrow$ $X$ given by $\pi(r, \theta)=\pi^{r}(\theta)=r \theta$ for all $r \in S$ and $\theta \in \mathbb{T}^{1}$. The semiflow $(X, S)$ is strongly transitive since $\overline{S^{-}(\theta)}=X$ for all $\theta \in X$. In fact, $S^{-}(\theta)=X$ or $S^{-}(\theta)=X \backslash\{0\}$. But $(X, S)$ is not minimal since $\{0\}$ is a minimal subset of $X$.

Also $\pi^{0} X=\{0 \cdot \theta: \theta \in X\}$ has empty interior and so $(X, S)$ is not almost open or open, yet $(X, S)$ is very strongly transitive.

In fact, $(X, S)$ is locally eventually onto and so also mixing and weak mixing.

For the Furstenberg family $\mathcal{F}$, the semiflow $(X, S)$ is called $\mathcal{F}$-strongly transitive if for every non-empty, open $U \in X$ and $x \in X$, one has $N_{S}(U, x) \in \mathcal{F}$.

Remark 2.51. If $(X, S)$ is $\mathcal{F}$ - strongly transitive then it is also $\mathcal{F}$-transitive.

Remark 2.52. Note that if $(X, S)$ is $\mathcal{F}$ - strongly transitive then $(X, S)$ is also $\mathcal{F}$-strongly central and $\widehat{\Omega}_{\mathcal{F}}(S)=X$.

2.6. Strongly Product Transitive. We note that transitivity is not preserved by taking products. One can just consider the irrational rotation on $\mathbb{T}$ as an example. So it becomes a natural question as to when can transitivity be preserved under products. This leads to the concept of weak mixing in topological dynamics.

What can be said about strongly transitive systems?

Example 2.53. Consider a modification of Example 2.6, where we consider

$$
S=\left\{f_{p, q}(x):=p x+q(\bmod 1): p \in \mathbb{D}, q \in \mathbb{D}\right\}
$$

with $\mathbb{D}=\left\{\frac{m}{2^{n}}: n \in \mathbb{N}\right.$ and $\left.0 \leq m \leq 2^{n}\right\}$ and operation as composition of functions. The monoid $S$ acts on $X$ and $(X, S)$ is a semiflow.

For the semiflow $(X, S)$, we have $\overline{S x}=X$ for all $x \in X$ and so $(X, S)$ is minimal. Note that $(X, S)$ is neither central, nor is Abelian.

Also, $(X, S)$ is strongly transitive since $X=\bigcup_{s \in S} s U$, for every open $U \subset X$.

Here $S^{-}(0,0)$ is not dense in $X \times X$, and so the product semiflow $(X \times X, S)$ fails to be be strongly transitive.

This leads to the concept of "strongly product transitive". We note that strongly product transitive systems were first defined and studied in [2]. We extend that study here.

We recall the following from [2]: 
$(X, f)$ is called Strongly Product Transitive if for every positive integer $k$ the product system $\left(X^{k}, f^{(k)}\right)$ is strongly transitive.

Note that for homeomorphisms, minimality is equivalent to strongly transitive, and hence homeomorphisms can never be strongly product transitive.

We recall the properties of strongly product transitive semicascades from 2 .

We recall the concept of multi-transitivity. $(X, f)$ is called multi-transitive if for each $m \in \mathbb{N},\left(X^{m}, f \times f^{2} \times \ldots \times f^{m}\right)$ is transitive [16]. While weakly mixing means weak disjointness of $f$ with itself, multi-transitivity means weak disjointness of $f$ with its iterates. Kwietniak and Oprocha 14 have shown that in general there is no connection between weak mixing and multi-transitivity citing examples of spacing subshifts which possess just one of these properties, though they are equivalent for minimal cascades. Also weakly mixing (semi)cascades which are very strongly transitive are multi-transitive.

$(X, f)$ is called multi-minimal if for each $m \in \mathbb{N}$, the system $\left(X^{m}, f \times f^{2} \times\right.$ $\left.\ldots \times f^{m}\right)$ is minimal. Examples of multi-minimal systems are discussed in 14. We see that the concept of multi-minimality and strongly product transitive are very distinct, since multi-minimality implies that $\left(X^{m}, f \times f^{2} \times \ldots \times f^{m}\right)$ is strongly transitive whereas for strongly product transitive we require $\left(X^{m}, f^{(m)}\right)$ is strongly transitive.

Definition 2.54. A semiflow $(X, S)$ is called Strongly Product Transitive if for every $k \in \mathbb{N}$ the product system $\left(X^{k}, S\right)$ is strongly transitive.

Remark 2.55. Consider the semiflow $(X, S)$. Note that for any non-empty, open $U_{1}, U_{2}, \subset X$ and $x, y \in X$, we have $N_{S}\left(U_{1} \times U_{2}, x \times y\right)=N_{S}\left(U_{1}, x\right) \cap N_{S}\left(U_{2}, y\right)$.

If $U \cap V=\emptyset$, then $N_{S}(U, x) \cap N_{S}(V, x)=\emptyset$. Thus, we cannot have something like Furstenberg Intersection lemma for these hitting sets.

We can consider the transitive semicascade on an interval $(I, f)$, with $\left(I, f^{2}\right)$ not transitive. Look for such examples and their properties in [17. Then we have open intervals $U, V \subset I$, such that $f(U)=V$ and $f(V)=U$. Thus for any $x \in U$, $N(U, x)$ will comprise of even entries and $N(V, x)$ will comprise of odd entries.

Proposition 2.56. For semiflow $(X, S)$ the following are equivalent:

(1) $(X, S)$ is strongly product transitive.

(2) The collection $\left\{N_{S}(U, x): x \in X\right.$ for $U$ non-empty, open in $\left.X\right\}$ generates a filter of subsets of $S$.

The proof is trivial and follows from definition.

Corollary 2.57. Strongly product transitive semiflows are weakly mixing.

Proof. The proof follows since for $x \in V, N_{S}(U, x) \subset N_{S}(U, V)$.

Remark 2.58. If $(X, S)$ is locally eventually onto then it is strongly product transitive.

Let $\mathcal{F}$ be a Furstenberg family. A semiflow $(X, S)$ is called $\mathcal{F}$-Strongly Product Transitive if for every $k \in \mathbb{N}$ the product system $\left(X^{k}, S\right)$ is $\mathcal{F}$ - strongly transitive.

Remark 2.59. Note that for any non-empty, open $U_{1}, U_{2} \subset X$ and $x, y \in X$, since $N_{S}\left(U_{1} \times U_{2}, x \times y\right)=N_{S}\left(U_{1}, x\right) \cap N_{S}\left(U_{2}, y\right),(X, S)$ is $\mathcal{F}$ - strongly product transitive then $(X, S)$ is $\mathcal{F}$ - weakly mixing if and only if $\mathcal{F}$ has the finite intersection property. 
Proposition 2.60. Let $\mathcal{F}$ be a Furstenberg family such that $\mathcal{F} \cdot \mathcal{F}=\mathcal{F}$. If $S$ is Abelian, $(X, S)$ is $\mathcal{F}$-weakly mixing and $\mathcal{F}$-strongly central then $(X, S)$ is $\mathcal{F}$ strongly product transitive.

Proof. Let $x, y \in X$ and open $U, V \subset X$ be such that $x \in U$ and $y \in V$. For any non-empty, open $A, B \subset X$, we see that $N_{S}(A \times B, U \times V) \in \mathcal{F} \cdot \mathcal{F}=\mathcal{F}$ and $N_{S}(U \times V, x \times y)=N_{S}(U, x) \cap N_{S}(V, y) \in \mathcal{F}$.

Now $N_{S}(A \times B, x \times y)=N_{S}(A \times B, U \times V) \cdot N_{S}(U \times V, x \times y)$ and so is in $\mathcal{F}$.

Without loss of generality, this can be extended to any finite product.

\section{SARANSH}

We have these results for semiflows similar to the results for semicascades in 2 . We skip their trivial proofs.

Theorem 3.1. Let $\phi:(X, T) \rightarrow(Y, T)$ be a factor map of dynamical systems.

If $(X, T)$ is strongly transitive, very strongly transitive, strongly product transitive or locally eventually onto then $(Y, T)$ satisfies the corresponding property.

Theorem 3.2. We have the following:

(a) If $(X \times Y, T \times S)$ is strongly transitive, strongly product transitive or locally eventually onto then both $(X, T)$ and $(Y, S)$ satisfy the corresponding property.

(b) Assume $(Y, S)$ is mixing. If $(X, T)$ topologically transitive, weak mixing, or mixing then $(X \times Y, T \times S)$ satisfy the corresponding property.

(c) Assume $(Y, S)$ is locally eventually onto. If $(X, T)$ is strongly transitive, very strongly transitive, strongly product transitive or locally eventually onto then $(X \times Y, T \times S)$ satisfies the corresponding property.

All these properties defined above are related.

$\begin{aligned} \text { Locally Eventually Onto } & \Longrightarrow \text { Mixing } \\ & \text { Strongly Product Transitive } \Longrightarrow \text { Weak Mixing } \Longrightarrow \text { Transitive }\end{aligned}$

Minimal $\Longrightarrow$ Transitive $\Longleftarrow$ Strongly Transitive $\Longleftarrow$ Very Strongly Transitive $\Longleftarrow$ Locally Eventually Onto

Moreover,

Minimal $\stackrel{\text { Abelian }+ \text { Central }}{\Longrightarrow}$ Very Strongly Transitive $\Longrightarrow$ Strongly Transitive

The reverse implications do not hold here.

We try to look into those transitivies which are not compared in the above relationship implications.

1. Locally Eventually Onto vs Minimality: Consider the one-sided shift system. This semicascade is locally eventually onto but not minimal. Again Example 2.6 gives a Minimal semiflow which is not Locally Eventually Onto.

2. Minimality vs Strongly Product Transitive: Again the one-sided shift system gives a Strongly Product Transitive semicascade which is not minimal. Whereas Example 2.53 is a Minimal semiflow which is not Strongly Product Transitive.

In fact, more can be said here. Since minimality is equivalent to strongly transitive for flows and cascades, and product of minimal systems is not minimal - these systems can never be strongly product transitive. For semicascades, minimality is almost one-to-one and guarantees the existence of an invariant subsytem which is a cascade. And so such systems cannot be strongly product transitive.

Can we have a minimal semiflow that is strongly product transitive? 
The answer is affirmative which can be seen in Example 3.3 below.

3. Strongly Product Transitive vs Mixing: The one-sided shift system gives a Mixing cascade which is not Strongly Product Transitive.

What can be said conversely?

Strongly Product Transitive systems are always weakly mixing. And so if there exists a counterexample here, we are looking out for the possibility of a weakly mixing system which is not mixing. If there exists no such counterexample, then we have a possibility that strongly product transitivity will always imply mixing.

\section{This is one of the unsolved problems mentioned in [2]}

For the case of cascades, we rule out any homeomorphic example. But all known examples of weakly mixing but not mixing cascades are (minimal)homeomorphisms and we are not sure if a non homeomorphic (non minimal) case would exist. It remains still open whether a semicascade example can be found in this case.

Again, we cannot have such an example for flows. What happens in case of semiflows? We have an affirmative answer here in form of Example 3.3 below.

Example 3.3. Define $\tau_{q}: \mathbb{T} \rightarrow \mathbb{T}$ as $\tau_{q}(\theta)=q \theta(\bmod 1), q \in \mathbb{Q}$ with $q \geq 2$ and $\mu: \mathbb{T} \rightarrow \mathbb{T}$ as $\mu(\theta)=\theta+\alpha(\bmod 1)$ where $\alpha \in\left(0,2^{-100}\right)$ is irrational. Then $\left\{\tau_{q}: q \in \mathbb{Q}, q \geq 2\right\} \cup\left\{\mu^{m}: m \in \mathbb{Z}\right\}$ generates a semigroup $S$ of continuous functions on $\mathbb{T}$ endowed with the uniform topology and with the operation of composition of functions. We see that $S$ is not Abelian.

Consider the semiflow $(\mathbb{T}, S)$. Note that $(\mathbb{T}, S)$ is central.

Also $S \theta$ is dense in $\mathbb{T}$ for all $\theta \in \mathbb{T}$. Thus $(\mathbb{T}, S)$ is minimal.

Consider $S^{-}(\theta)$. We note that $S^{-}(\theta)$ is dense in $\mathbb{T} \forall \theta \in \mathbb{T}$ and hence $(\mathbb{T}, S)$ is strongly transitive.

Again for given non-empty, open $U \subset X$, there is a finite $K \subset\left\{\mu^{m}: m \in \mathbb{Z}\right\}$, for which $\mathbb{T}=\bigcup_{s \in K} s U$. Thus $(\mathbb{T}, S)$ is very strongly transitive.

Furthermore, we observe that for every non-empty, open $U \subset \mathbb{T}$ there exists a $L \in \mathbb{Q}, L \geq 2$ such that $\tau_{l}(U)=\mathbb{T}$ for all $l \geq L$. Hence for all $n \in \mathbb{N}$ and non-empty, open $U_{1}, \ldots, U_{n} \subset \mathbb{T}$ there exists $M_{n} \in \mathbb{Q}$ for which $\tau_{M_{n}}\left(U_{1} \times \ldots \times U_{n}\right)=\mathbb{T}^{n}$. This validates that $(\mathbb{T}, S)$ is strongly product transitive.

We take open $U, V \subset \mathbb{T}$ with diameter less than $\alpha$ such that $\frac{\pi}{2} \in U$ and $\frac{3 \pi}{2} \in V$. Then there exists $P \in \mathbb{Q}$ such that $\tau_{p}(U) \cap V=\emptyset$, for $p \in[2, P] \cap \mathbb{Q}$.

Suppose $(\mathbb{T}, S)$ is mixing. Then for the non-empty, open $U, V \subset \mathbb{T}$ taken above, there exists a compact $K \subset S$ for which $\lambda(U) \cap V \neq \emptyset$ for all $\lambda \in S \backslash K$. Clearly, $\left\{\tau_{p}: p \in[2, P] \cap \mathbb{Q}\right\} \subset K$. Let $i \in[2, P] \cap(\mathbb{R} \backslash \mathbb{Q})$ be such that $p_{n} \rightarrow i$ for a sequence $\left\{p_{n}\right\}$ with $p_{n} \in[2, P] \cap \mathbb{Q}$. Consider $\tau_{i}: \mathbb{T} \rightarrow \mathbb{T}$ as $\tau_{i}(\theta)=i \theta(\bmod 1)$. Then $\tau_{p_{n}} \rightarrow \tau_{i}$, but $\tau_{i} \notin K$ contradicting the compactness of $K$.

Hence $(\mathbb{T}, S)$ is not mixing.

\section{REFERENCES}

[1] Ethan Akin, Recurrence in topological dynamics: Furstenberg families and Ellis actions, The University Series in Mathematics. Plenum Press, New York, (1997).

[2] Ethan Akin, Joseph Auslander and Anima Nagar, Variations on the Concept of Topological Transitivity, Studia Math. 235 (3) (2016), 225-249.

[3] Joseph Auslander, Minimal flows and their extensions, North-Holland Mathematics studies,153 (1988).

[4] Joseph Auslander and Xiongping Dai, Minimality, distality and equicontinuity for semigroup actions on compact Hausdorff spaces, Discrete \& Continuous Dynamical Systems - A, 39(2019) 4647-4711. 
[5] G. Cairns, A. Kolganova, A. Nielsen, Topological transitivity and mixing notions for group actions. Rocky Mt. J. Math. 37(2007), No. 2, 371-397.

[6] Hillel Furstenberg, Disjointness in ergodic theory, minimal sets and a problem in diophantine approximation, Mathematical Systems theory, 1 (1967), 1-49.

[7] Shmuel Glasner, Proximal flows, Springer Berlin Heidelberg, (1976).

[8] Eli Glasner, Ergodic theory via joinings, AMS, Surveys and Monographs, 101 (2003).

[9] W. H. Gottschalk and G. A. Hedlund, The Dynamics Of Transformation Groups, Trans. of the Amer. Math. Soc., 65, 348-359 (1949).

[10] W. H. Gottschalk and G. A. Hedlund, Topological dynamics, Amer. Math. Soc. Colloquium Publications, vol. 36, 1955.

[11] Anzelm Iwanik, Independent sets of transitive points, Banach Center Publications, 23(1989), $277-282$.

[12] Sergiy Kolyada, and Lubomir Snoha Some aspects of topological transitivity - a survey, Grazer Math. Ber., 334, 3-35(1997).

[13] Sergiy Kolyada, Lubomir Snoha, and Sergei Trofimchuk, Noninvertible Minimal Maps, Fundamenta Mathematicae. 168 , 141-163(2001).

[14] Dominik Kwietniak and Piotr Oprocha, On weak mixing, minimality and weak disjointness of all iterates, Ergod. Th. \& Dynam. Sys. 32 (2012), 1661-1672.

[15] Jian Li, Transitive points via Furstenberg family, Topology and its Applications, 158 (2011) 2221-2231.

[16] T.K.S. Moothathu, Diagonal points having dense orbit, Colloq. Math., 120 (2010), 127-138.

[17] Anima Nagar and V. Kannan, Topological Transitivity for Discrete Dynamical Systems, Applicable Mathematics In The Golden Age, Narosa Publications(2003), 534-584.

[18] Jan de Vries, Elements of Topological Dynamics, Mathematics and its Applications 257, Kluwer, Dordrecht, (1993).

Department of Mathematics, Indian Institute of Technology Delhi, Hauz Khas, New DELHi 110016, INDIA 